University of Nebraska - Lincoln

DigitalCommons@University of Nebraska - Lincoln

8-2-2001

\title{
Coexistence of covalent and metallic bonding in the boron intercalation superconductor $\mathrm{MgB}_{2}$
}

Kirill D. Belashchenko

University of Nebraska-Lincoln, belashchenko@unl.edu

Mark van Schilfgaarde

Sandia National Laboratories, mark.van_schilfgaarde@kcl.ac.uk

Vladimir P. Antropov

lowa State University, antropov@ameslab.gov

Follow this and additional works at: https://digitalcommons.unl.edu/cmrafacpub

Part of the Nanoscience and Nanotechnology Commons

Belashchenko, Kirill D.; van Schilfgaarde, Mark; and Antropov, Vladimir P., "Coexistence of covalent and metallic bonding in the boron intercalation superconductor $\mathrm{MgB}_{2}$ " (2001). Faculty Publications from Nebraska Center for Materials and Nanoscience. 22.

https://digitalcommons.unl.edu/cmrafacpub/22

This Article is brought to you for free and open access by the Materials and Nanoscience, Nebraska Center for (NCMN) at DigitalCommons@University of Nebraska - Lincoln. It has been accepted for inclusion in Faculty Publications from Nebraska Center for Materials and Nanoscience by an authorized administrator of DigitalCommons@University of Nebraska - Lincoln. 


\title{
Coexistence of covalent and metallic bonding in the boron intercalation superconductor $\mathrm{MgB}_{2}$
}

\author{
K. D. Belashchenko, ${ }^{1}$ M. van Schilfgaarde,${ }^{2}$ and V. P. Antropov ${ }^{1}$ \\ ${ }^{1}$ Ames Laboratory, Iowa State University, Ames, Iowa 50011 \\ ${ }^{2}$ Sandia National Laboratories, Livermore, California 94551
}

(Received 16 February 2001; published 7 August 2001)

\begin{abstract}
Chemical bonding and electronic structure of $\mathrm{MgB}_{2}$, a boron-based newly discovered superconductor, is studied using self-consistent band-structure techniques. Analysis of the transformation of the band structure for the hypothetical series of graphite-primitive graphite-primitive graphitelike boron-intercalated boron, shows that the band structure of $\mathrm{MgB}_{2}$ is graphitelike, with $\pi$ bands falling deeper than in ordinary graphite. These bands possess a typically delocalized and metallic, as opposed to covalent, character. The in-plane $\sigma$ bands retain their two-dimensional (2D) covalent character, but exhibit a metallic hole-type conductivity. The coexistence of 2D covalent in-plane and three-dimensional (3D) metallic-type interlayer conducting bands is a peculiar feature of $\mathrm{MgB}_{2}$. We analyze the 2D and 3D features of the band structure of $\mathrm{MgB}_{2}$ and related compounds, and their contributions to conductivity.
\end{abstract}

DOI: $10.1103 /$ PhysRevB.64.092503

PACS number(s): 74.25.Jb, 71.20.-b, 72.15.-v

The recent discovery of superconductivity (SC) in $\mathrm{MgB}_{2}$ (Ref. 1) has stimulated significant interest in this system.,3 One of the first questions is whether $\mathrm{MgB}_{2}$ represents a new class of superconductors, or whether it is related to other known classes in terms of its bonding and electronic properties. The crystal structure of $\mathrm{MgB}_{2}$ may be regarded as that of completely intercalated graphite ${ }^{4}$ with carbon replaced by boron (its neighbor in the Periodic Table). In addition, $\mathrm{MgB}_{2}$ is formally isoelectronic to graphite. Therefore, understanding the bonding and electronic properties of $\mathrm{MgB}_{2}$ and its relation to graphite intercalation compounds (GIC's), some of which also exhibit SC, is desirable and is the subject of this paper.

The search for high-temperature superconductivity in carbon phases started in 1965 when $T_{c}=0.55 \mathrm{~K}$ in $\mathrm{KC}_{8}$ was reported, and $\mathrm{SC}$ was subsequently explained in terms of the interaction of $\pi$-derived bands with the longitudinal phonon modes of the graphite layer. ${ }^{5}$ Similar conclusions for the electronic structure at the Fermi level were derived for another GIC, $\mathrm{LiC}_{6}$. The highest $T_{c}$ achieved for a GIC was 5 K. ${ }^{6}$ A parallel development was that of SC in Bechgard salts, the organic charge-transfer systems. Within this family of organic materials exhibiting carbon $\pi$-band conductivity, $T_{c}$ has been raised to $12.5 \mathrm{~K}^{7}$ It should be noted that the majority of these compounds are unstable at normal conditions and high-pressure experiments are most common. This part of GIC studies was reviewed in Ref. 5.

Later research shifted to the area of carbides and nitrides of transition metals where metallicity and conductivity are mostly due to transition-metal atoms. Only in 1991 was it shown that alkali-doped $\mathrm{C}_{60}$ exhibits $\mathrm{SC}$ with maximum $T_{c}$ of $33 \mathrm{~K}^{8}$ It was the first representative of a threedimensional (3D) carbon-based metal with a modified (compared to graphite) coupling of lattice vibrations to the conduction electrons due to the curvature of the molecule but still with $\pi$-band derived states at the Fermi level.

We have calculated the electronic structure of $\mathrm{MgB}_{2}$ and some related compounds using the Stuttgart linear muffin-tin orbital tight-binding in atomic sphere approximation (ASA) code and full-potential LMTO (FLMTO) method. ${ }^{9}$ A local exchange-correlation potential was used in both cases (the addition of gradient corrections did not change the results). It appears that a general analysis of energy bands may be safely done in ASA, while a reliable treatment of charge densities and anisotropy of transport properties of these compounds requires more accurate FLMTO calculations.

In order to examine the relation between the band structure of $\mathrm{MgB}_{2}$ and that of graphite we studied the following hypothetical sequence of intermediate materials: carbon in the "primitive graphite" (PG) lattice with no displacement between layers as in $\mathrm{MgB}_{2}$ and in GIC's, using graphite lattice parameters; boron in the PG lattice with $a$ as in $\mathrm{MgB}_{2}$ and $c / a$ as in graphite; boron in the PG lattice with $a$ and $c / a$ as in $\mathrm{MgB}_{2} ; \mathrm{LiB}_{2}$ in the same structure; $\mathrm{MgB}_{2}$ itself. The results of some of these calculations are shown in Fig. 1. The band structure of PG carbon shown in Fig. 1(a) is very similar to that of graphite (see, e.g., Ref. 10 and references therein) with the appropriate zone folding for a smaller unit cell. (This is quite natural because of the weak interlayer interaction.) Boron in the same lattice but scaled to match the $\mathrm{MgB}_{2}$ in-plane lattice parameter (not shown) has nearly identical bands with the energies scaled by the inverse square of the lattice parameter, in agreement with common tightbinding considerations. ${ }^{11}$ Figure $1(\mathrm{~b})$ shows the natural enhancement of the out-of-plane dispersion of the $\pi$ bands when the interlayer distance is reduced. Figures $1(\mathrm{c})$ and $1(\mathrm{~d})$ demonstrate that "intercalation" of boron by $\mathrm{Li}$ or Mg produces a significant distortion of the band structure, so that the role of the intercalant is not simply one of donating electrons to boron's bands [which would return the band structure to that of PG carbon shown in Fig. 1(a)]. The main change upon intercalation is the downward shift of the $\pi$ bands compared to $\sigma$ bands. For Li this shift of $\sim 1.5 \mathrm{eV}$ is almost uniform throughout the Brillouin zone. Replacement of Li by $\mathrm{Mg}$ shifts the $\pi$ bands further, but this shift is strongly asymmetric increasing from $\sim 0.6 \mathrm{eV}$ at the $\Gamma$ point to $\sim 2.6 \mathrm{eV}$ at the $A$ point. In addition, the out-of-plane dispersion of the $\sigma$ bands is also significantly enhanced. $\mathrm{In}_{\mathrm{LiB}}$ the filling of the bonding $p_{x y}$ bands is nearly the same as in PG boron, 

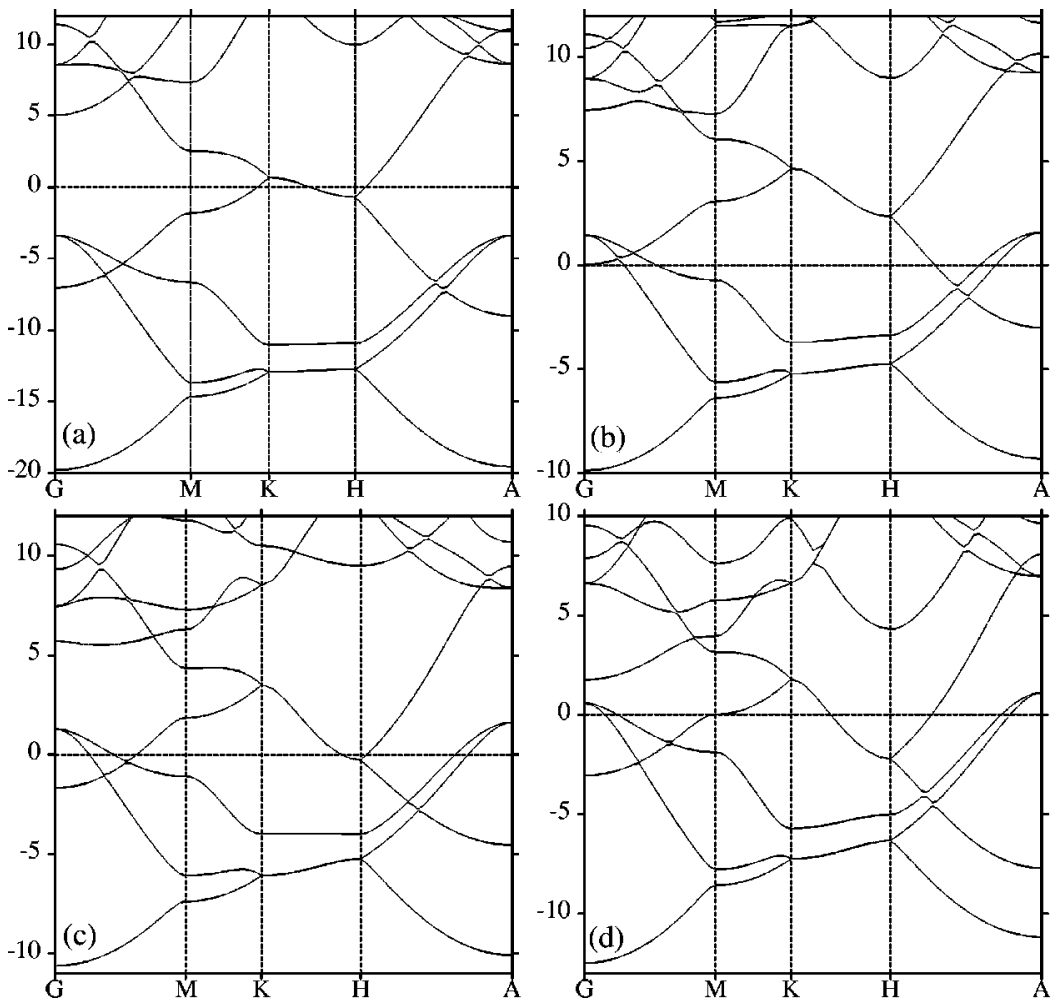

FIG. 1. Band structures of (a) primitive ( $A A$ stacking) graphite (PG), $a=2.456 \AA, c / a$ $=1.363 ;$ (b) PG boron, $a=3.085 \AA, c / a$ $=1.142$ (as in $\mathrm{MgB}_{2}$ ); (c) $\mathrm{LiB}_{2}$ in $\mathrm{MgB}_{2}$ structure, same $a$ and $c / a$; (d) $\mathrm{MgB}_{2}$, same $a$ and $c / a$. Energy is in $\mathrm{eV}$ relative to $E_{F}$. The order of occupied bands in the $\Gamma$ point is $\sigma$ bonding with boron $s$ character, $\pi$ bonding with boron $p_{z}$ character, and $\sigma$ bonding with boron $p_{x y}$ character (double degenerate). while in $\mathrm{MgB}_{2}$ the Fermi level shifts closer to the top of these bands.

The Fermi surface (FS) for $\mathrm{MgB}_{2}$ obtained in the FLMTO method is practically identical to Fig. 3 of Ref. 2. The incompletely filled bonding $\sigma$ bands with predominantly boron $p_{x y}$ character form two hole-type cylinders around the $\Gamma-A$ line, while the $\pi$ bands form two planar honeycomb tubular networks: one electron-type network centered at $k_{z}=0$ and another similar, but more compact, hole-type network centered at $k_{z}=\pi / c$. The lowering of the $\pi$ band in $\mathrm{MgB}_{2}$ compared to the PG boron is connected with the charge transfer from magnesium to the interstitial region. The increased charge density in the interstitials also enhances the overlap of the boron $p_{z}$ orbitals and hence the $k_{z}$ dispersion.

The nature of bonding in $\mathrm{MgB}_{2}$ may be understood from the charge density (CD) plots shown in Fig. 2. As seen in Fig. 2(a), bonding in the boron layer is typically covalent. The CD of the boron atom is strongly aspherical, and the directional bonds with high $\mathrm{CD}$ are clearly seen (see also Ref. 12). It is worth noting that the CD distribution in the boron layer is very similar to that in the carbon layer of graphite (see, e.g., Ref. 10). This directional in-plane bonding is also obvious from Fig. 2(b) showing the CD in a cross section containing both $\mathrm{Mg}$ and $\mathrm{B}$ atoms. However, Fig. 2(b) also shows that a large amount of valence charge does not participate in any covalent bonding, but rather is distributed more or less homogeneously over the whole crystal. Further, Fig. 2(c) shows the difference of the $\mathrm{CD}$ of $\mathrm{MgB}_{2}$ and that of hypothetical $\mathrm{NaB}_{2}$ in exactly the same lattice. Not only does it show that one extra valence electron is not absorbed by boron atoms but is rather delocalized in the interstitials; it also shows that some charge moves outward from boron atoms and covalent in-plane B-B bonds. Figure 2(d) shows the
$\mathrm{CD}$ difference between the isoelectronic compounds $\mathrm{MgB}_{2}$ and $\mathrm{PG}$ carbon $\left(\mathrm{C}_{2}\right)$. In $\mathrm{MgB}_{2}$ the electrons see approximately the same external potential as in $\mathrm{C}_{2}$, except that one proton is pulled from each $\mathrm{C}$ nucleus and put at the $\mathrm{Mg}$ site. It is evident that the change $\mathrm{C}_{2} \rightarrow \mathrm{MgB}_{2}$ weakens the twocenter $\sigma$ bonds (the charge between the atoms is depleted) and redistributes it into a delocalized, metallic density.

Thus, the structure of $\mathrm{MgB}_{2}$ is held together by strongly covalent bonding in boron sheets and by delocalized, metallic-type bonding between these sheets. A peculiar feature of this compound is that electrons participating in both of these bond types provide comparative contributions to the density of states (DOS) at the Fermi level (see below). This distinguishes $\mathrm{MgB}_{2}$ from closely related GIC's where covalent bonds in the carbon sheets are always completely filled, while the nearly cylindrical parts of the FS commonly found in those compounds are formed by carbon-derived $\pi$ bands that are also responsible for conductivity in pristine graphite. $^{5}$

Because of the coexistence of two different types of chemical bonds, it is desirable to find the contributions to the total DOS and transport properties from separate pieces of the FS originating from two-dimensional (2D) covalent and 3D metallic-type bonding. Such decomposition is shown in Fig. 3 for the total DOS and for the in-plane $(x x)$ and outof-plane $(z z)$ components of the tensor $\sigma_{\alpha \beta}$ $=\int v_{\alpha} v_{\beta} \delta\left[\varepsilon(\mathbf{k})-E_{F}\right] d \mathbf{k}$, with $v_{\alpha}$ being a component of the electronic velocity. One can see that the $3 \mathrm{D}$ (metallic-type bonding) and cylindrical (covalent bonding) parts of the FS contribute, respectively, about $55 \%$ and $45 \%$ to $N(0) . N(\varepsilon)$ for the hole-type zones rapidly decreases with increasing $\varepsilon$ and already at $\varepsilon \approx 0.8 \mathrm{eV}$ above $E_{F}$ the total DOS is almost 

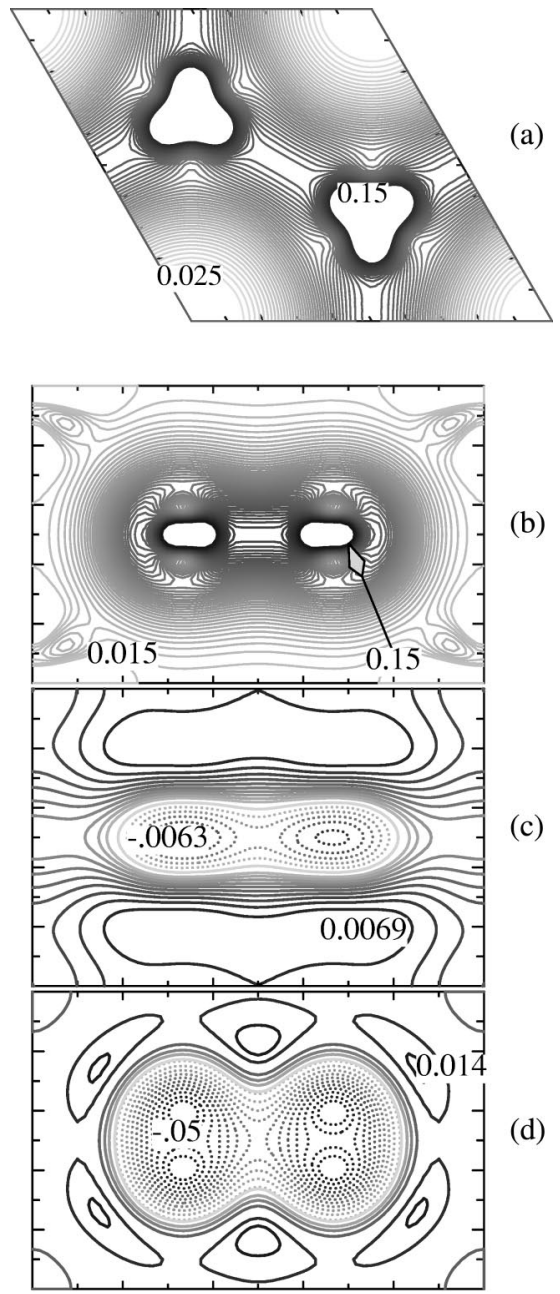

(b)

(c)

(d)

FIG. 2. Pseudocharge density contours obtained in FLMTO. The unit cell is everywhere that of $\mathrm{MgB}_{2}$. The darkness of lines increases with density. (a) $\mathrm{MgB}_{2}$ in the (0002) plane passing through $\mathrm{B}$ nuclei; (b) $\mathrm{MgB}_{2}$ in the (1000) plane passing through $\mathrm{Mg}$ nuclei at each corner of the figure. B nuclei occupy positions $(1 / 3,1 / 2)$ and $(2 / 3,1 / 2)$ in the plane of the figure. The integrated charge of the unit cell is 8. (c) The (1000) plane, difference in smoothed density, $\mathrm{MgB}_{2}$ minus $\mathrm{NaB}_{2}$. The integrated charge of the unit cell is 1. (d) The (1000) plane, difference in smoothed density, $\mathrm{MgB}_{2}$ minus PG carbon. The integrated charge of the unit cell is 0 . In (a) and (b) the regions with high charge densities close to the nuclei are not plotted. In (c) and (d), dotted lines show negative values.

completely determined by the 3D electron-type band. The latter contribution is almost constant and probably is not related to the change of SC properties under pressure or with doping. The corresponding contribution to $\sigma$ exceeds all other contributions (more than $50 \%$ for $\sigma_{z z}$ and $\sigma_{x x}$ ) and is virtually isotropic. Holes in the in-plane B-B covalent bands (two cylinders at the FS) as expected have clearly anisotropic behavior contributing about $30 \%$ to $\sigma_{x x}$ and virtually nothing to $\sigma_{z z}$. The 3D hole-type part of the FS is also notably anisotropic with predominantly $z$-axis conductivity. The total $\sigma$ has a rather small anisotropy at $E_{F}$ with $\sigma_{x x} / \sigma_{z z} \simeq 1.22$. The average projections of the Fermi velocities $\bar{v}_{\alpha}=\left\langle v_{\alpha}^{2}\right\rangle^{1 / 2}$ are $\bar{v}_{x}=5.36 \times 10^{7} \mathrm{~cm} / \mathrm{s}, \bar{v}_{z}=4.85 \times 10^{7} \mathrm{~cm} / \mathrm{s}$. The average

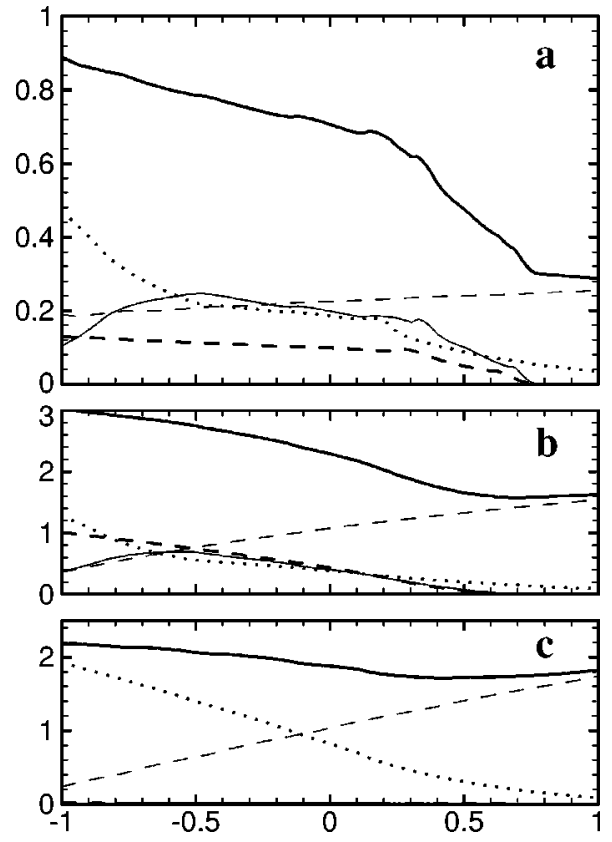

FIG. 3. Rigid band results for $\mathrm{MgB}_{2}$ : (a) total DOS in $\mathrm{eV}^{-1} /$ cell; (b) $\sigma_{x x}$ and (c) $\sigma_{z z}$ in Ry $a_{0}^{2}$ (for a definition of $\sigma_{\alpha \beta}$ see text), with contributions from different parts of the FS. Thick solid lines: total; thin dashed lines: 3D electronic part; dotted lines: 3D hole part; thick dashed lines: internal cylinder; thin solid lines: external cylinder. Energy is in $\mathrm{eV}$ relative to $E_{F}$.

Fermi velocities $\bar{v}_{\nu}=\left\langle v^{2}\right\rangle_{\nu}^{1 / 2}$ for each Fermi-surface sheet $\nu$ are as follows: internal cylinder, $8.85 \times 10^{7} \mathrm{~cm} / \mathrm{s}$; external cylinder, $6.02 \times 10^{7} \mathrm{~cm} / \mathrm{s} ; 3 \mathrm{D}$ electronic sheet, 1.12 $\times 10^{8} \mathrm{~cm} / \mathrm{s}$; 3D hole sheet, $8.74 \times 10^{7} \mathrm{~cm} / \mathrm{s}$.

The main challenge is to suggest another possible SC with a higher $T_{c}$. We studied several similar systems that, as we believe, may be interesting for experimental studies. Several isoelectronic compounds demonstrate very different behavior. Hypothetical $\mathrm{BeB}_{2}$ and $\mathrm{ZnB}_{2}$ in the $\mathrm{MgB}_{2}$ structure are very similar to $\mathrm{MgB}_{2}$ in terms of the electronic properties. Our ASA calculations of these compounds with lattice parameters of $\mathrm{MgB}_{2}$ produced very similar band structures with nearly identical FS. Presumably a smaller radius of the $\mathrm{Be}$ ion and a larger one of the $\mathrm{Zn}$ ion will generate some difference but we expect that these compounds (if existing) would have properties similar to $\mathrm{MgB}_{2}$. Another isoelectronic system is lithium borocarbide, LiBC, ${ }^{13}$ which, according to our ASA results, is a perfect insulator, so that any substitution of $\mathrm{C}$ by $\mathrm{B}$ will lead to metallic behavior. (In general, the obtained band structure of this system is very similar to that of $\mathrm{BN} .^{14}$ ) Experimentally very small conductivity was observed in LiBC. ${ }^{13}$

The addition of one electron to $\mathrm{MgB}_{2}$ corresponds to $\mathrm{AlB}_{2}$. This compound also has metallic conductivity but its FS does not have any cylindrical parts as in $\mathrm{MgB}_{2}$. Experimentally no $T_{c}$ has been observed ${ }^{15}$ which makes electron doping an unlikely mechanism to increase $T_{c}$. Under such circumstances it would be most logical to try hole doping by replacing $\mathrm{Mg}$ by $\mathrm{Na}$ or $\mathrm{Li}$. Unfortunately such compounds 
are unstable. We are familiar only with one experimental report that $\mathrm{NaC}_{2}$ (Ref. 16) is a weak $\mathrm{SC}$ under pressure and is very unstable. However, it may be possible to form an alloy $(\mathrm{Mg}, \mathrm{Na}) \mathrm{B}_{2}$ with modest amounts of $\mathrm{Na}$. It is evident from Fig. 3 that the hole-type parts of the FS change dramatically with electron filling. More relevant discussions can be found in Ref. 17.

In summary, $\mathrm{MgB}_{2}$ represents the first relatively high- $T_{c}$ superconducting compound of boron and has very peculiar bonding characteristics compared to GIC. We showed that the electronic structure of $\mathrm{MgB}_{2}$ has both similarity to and notable differences with the GIC's. These features provide a basis both for further studies of normal
andSC states of $\mathrm{MgB}_{2}$ and for engineering new SC compounds.

The authors acknowledge useful discussions with $\mathrm{S}$. Bud'ko, P. Canfield, K. Ho, I. Mazin, and G. Miller. This work was carried out at the Ames Laboratory, which is operated for the U.S. Department of Energy by Iowa State University under Contract No. W-7405-82. This work was supported by the Director for Energy Research, Office of Basic Energy Sciences of the U.S. Department of Energy. M.V.S. was supported by the Office of Basic Energy Sciences of the U.S. DOE, Division of Materials Science under Contract No. DE-AC04-94AL85000.
${ }^{1}$ J. Nagamatsu, N. Nakagawa, T. Muranaka, Y. Zenitani, and J. Akimitsu, Nature (London) 410, 63 (2001).

${ }^{2}$ J. Kortus, I. I. Mazin, K. D. Belashchenko, V. P. Antropov, and L. L. Boyer, Phys. Rev. Lett. 86, 4656 (2001).

${ }^{3}$ S.L. Bud'ko, G. Lapertot, C.E. Petrovic, C. Cunningham, N. Anderson, and P.C. Canfield, Phys. Rev. Lett. 86, 1877 (2001).

${ }^{4}$ J.K. Burdett and G.J. Miller, Chem. Mater. 2, 12 (1989).

${ }^{5}$ N. B. Brandt, S. M. Chudinov, and Ya. G. Ponomarev, Modern Problems in Condensed Matter Sciences (North-Holland, Amsterdam, 1988), Vol. 20.1.

${ }^{6}$ I.T. Belash, A.D. Bronnikov, O.V. Zharikov, and A.V. Pal'nichenko, Synth. Met. 36, 283 (1990).

${ }^{7}$ H. Urayama, H. Yamochi, G. Saito, K. Nozawa, T. Sugan, and M. Kinoshita, Chem. Lett. 1988, 55 (1988).

${ }^{8}$ K. Tanigaki, T.W. Ebbesen, S. Saito, J. Muzuki, J.S. Tsai, Y. Kubo, and S. Kuroshima, Nature (London) 352, 222 (1991).

${ }^{9}$ M. Methfessel, M. van Schilfgaarde, and R. A. Casali, in Electronic Structure and Physical Properties of Solids: The Uses of the LMTO Method, edited by H. Dreysse, Lecture Notes in Physics Vol. 535 (Springer-Verlag, Berlin, 2000).

${ }^{10}$ H.J.F. Jansen and A.J. Freeman, Phys. Rev. B 35, 8207 (1987).

${ }^{11}$ W. A. Harrison, Electronic Structure and the Properties of Solids (Freeman, San Francisco, 1980).

${ }^{12}$ A.I. Ivanovskii and N.I. Medvedeva, Russ. J. Inorg. Chem. 45, 1234 (2000).

${ }^{13}$ M. Worle, R. Nesper, G. Mair, M. Schwarz, and H.G. von Schnering, Z. Anorg. Allg. Chem. 621, 1153 (1995).

${ }^{14}$ A. Catellani, M. Posternak, A. Baldereschi, H.J.F. Jansen, and A.J. Freeman, Phys. Rev. B 32, 6997 (1985).

${ }^{15}$ E. Sirtl and L.M. Woerner, J. Cryst. Growth 16, 215 (1972).

${ }^{16}$ I.T. Belash, A.D. Bronnikov, O.V. Zharikov, and A.V. Pal'nichenko, Solid State Commun. 69, 921 (1989).

${ }^{17}$ V. P. Antropov, K. D. Belashchenko, M. van Schilfgaarde, and S. N. Rashkeev, in Studies of High Temperature Superconductors, edited by A. V. Narlikar (Nova Science, New York, 2001). 